\title{
Gossypol inhibits the growth of MAT-LyLu prostate cancer cells by modulation of TGFB/Akt signaling
}

\author{
JIAHUA JIANG ${ }^{1}$, WEIPING YE ${ }^{2}$ and YOUNG C. LIN ${ }^{2,3}$ \\ ${ }^{1}$ Cancer Research Laboratory, Methodist Research Institute, Clarian Health Partners Inc., \\ 1800 N. Capitol Avenue, Indianapolis, IN 46202; ${ }^{2}$ Laboratory of Reproductive and Molecular Endocrinology, \\ College of Veterinary Medicine, ${ }^{3}$ The Ohio State University Comprehensive Cancer Center, \\ The Ohio State University, 1900 Coffey Road, Columbus, OH 43210, USA
}

Received September 30, 2008; Accepted November 26, 2008

DOI: 10.3892/ijmm_00000208

\begin{abstract}
Gossypol (GP), a male contraceptive compound naturally present in cottonseed products, possesses antiproliferative and anti-metastatic effects in vitro and in vivo. However, the detailed mechanisms responsible for the effects of GP on the cell cycle of prostate cancer cells remain to be elucidated. In the present study, we investigated the effects of GP on the regulation of the cell cycle of rodent prostate cancer MAT-LyLu cells and the mechanisms of GP-induced growth inhibition. Our results showed that GP inhibited the cell proliferation and colony formation in a dose-dependent manner by the up-regulation of expression and secretion of transforming growth factor $\beta 1$ (TGFß1) and down-regulation of expression of Akt and phospho-Akt protein. The inhibition of cell growth was also demonstrated by cell cycle arrest at G0/G1 phase. Furthermore, GP decreased the expression of cyclin D1, Cdk4 and phospho-Rb in MAT-LyLu cells. Thus, the inhibitory effects of GP on the proliferation of MAT-LyLu prostate cancer cells are associated with modulation of TGFß1 and Akt signaling, which influence the expression of regulatory proteins such as cyclin $\mathrm{D} 1, \mathrm{Cdk} 4$ and phospho- $\mathrm{Rb}$ which regulate cell cycle progression of prostate cancer cells.
\end{abstract}

\section{Introduction}

Prostate cancer is the most common malignancy and the second leading cause of male cancer-related death in the US, accounting for approximately $29 \%$ of all cancers diagnosed in males (1). The primary therapeutic strategies for the

Correspondence to: Dr Young C. Lin, Laboratory of Reproductive and Molecular Endocrinology, College of Veterinary Medicine, The Ohio State University, 1900 Coffey Road, Columbus, OH 432101092, USA

E-mail: lin.15@osu.edu

Key words: gossypol, cell cycle, transforming growth factor $\beta 1$, Akt, MAT-LyLu treatment of prostate cancer is androgen deprivation (2). Androgen withdrawal may initially reduce the growth of metastatic prostate cancers; however, the long-term treatment of prostate cancer patients results in loss of responsiveness. Prostate cancer cells finally become androgen independent, leading to the failure of androgen-deprivation therapy and patient death (2-4). Thus, it is necessary to look for new agents to treat androgen-independent prostate cancer.

Gossypol (GP) is a naturally occurring yellow pigment present in cottonseeds. It is well documented that GP inhibits the proliferation of many human cancer cells in vitro and in vivo (5-10). For example, GP significantly inhibited the growth of PC-3, DU-145, MCF-7 cells (6,8-10) as well as the in vivo growth and metastasis of prostate cancer MAT-LyLu cells after implantation into Copenhagen rats (5). GP induces cell cycle arrest at G0/G1 phase in human prostate cancer PC-3 cells (6) or induces apoptosis in PC-3 (11) and colon cancer HT-29 cells (12). (-)-GP, an enantiomer of GP, inhibits growth in breast cancer and prostate cancer cells as well as tumor growth of PC-3 cells in xenograft models (10,13-15).

Although the precise mechanism of action of GP remains unknown, it is believed that the anticancer effects of GP are related to apoptosis induction and cell cycle arrest at G0/G1 phase $(6,11,12,14)$. The molecular mechanism of GP-induced apoptosis is well defined. It was reported that GP induced apoptosis by inhibiting the heterodimerization of anti-apoptotic proteins $\mathrm{Bcl} 2 / \mathrm{Bcl}-\mathrm{xl}$ with pro-apoptotic molecules Bax and Bim in human prostate cancer PC-3 cells (11). In HT-29 colon cancer cells, GP induced apoptosis by interfering with the heterodimerization of Bcl-xl with another pro-apoptotic molecule Bak (12). GP was shown to induce apoptosis and cytochrome c release via a conformational change of $\mathrm{Bcl} 2$ in Bax-/Bak-embryonic fibroblast cells, and the activation of apoptosis was Bax/Bak independent (16). Furthermore, (-)-GP was observed to enhance the sensitivity of PC-3 tumors to radiation treatment through inhibiting the function of $\mathrm{Bcl}-\mathrm{xl}$ protein (15). However, the information on the mechanism of GP-induced cell cycle is limited. Experimental data suggest that GP may affect cell cycle distribution by modulating the expression of transforming growth factor $\beta 1$ (TGFß1) and cell cycle regulator cyclin D1 in prostate cancer cells $(6,9)$ suggesting the involvement of TGFß1 in GP-induced growth 
inhibition. TGFß1 has been shown to mediate the antiproliferative effects of many antitumor agents, including vitamin $\mathrm{D}_{3}(17)$, genistein (18), GP $(6,9)$ and tamoxifen (19). TGFß1 exerts its biological effects by binding to TGFß receptor II (TßRII), which induces formation of a heteromeric complex of TßRII and TßRI, triggering a signaling pathway that regulates cell cycle regulators such as $\mathrm{Rb}$, cyclin and cyclin-dependent kinase $(20,21)$. To date, the effect of GP on the cell cycle and TGFß1 in MAT-LyLu cells remains unknown. Moreover, TGFß1 has been recently reported to regulate the Akt signaling pathway in colon cancer cells, leading to inhibition of cell proliferation (22). Thus, we hypothesized that Akt may play an important role in GPinduced growth inhibition.

The objective of this study was to investigate the role of TGFß1 and Akt signaling in GP-induced growth inhibition of MAT-LyLu prostate cancer cells.

\section{Materials and methods}

Reagents. GP was purchased from Sigma (St. Louis, MO). GP was dissolved in $100 \%$ ethanol to make a $10-\mathrm{mM}$ stock solution. The solutions were aliquoted into glass vials and stored at $-20^{\circ} \mathrm{C}$. Treatment solutions were prepared by the dilution of stock solution in culture medium. Ultrapure natural human TGFß1 was purchased from Genzyme Corp. (Cambridge, MA).

Cell culture. MAT-LyLu cells were the generous gift from P. Ghosh of Ohio State University, who originally obtained the cells through the courtesy of Dr J.T. Isaacs, Johns Hopkins University. Cells were cultured in RPMI-1640 medium (Invitrogen, Grand Island, NY) containing an antibiotic-antimycotic mixture $(100 \mathrm{IU} / \mathrm{ml}$ penicillin, $100 \mu \mathrm{g} / \mathrm{ml}$ streptomycin and $0.25 \mu \mathrm{g} / \mathrm{ml}$ amphotericin) (Invitrogen, Carlsbad, CA) and 5\% fetal calf serum (FCS; Atlanta Biologicals, Norcross, GA) in a humidified incubator $\left(37^{\circ} \mathrm{C}, 5 \% \mathrm{CO}_{2}\right.$ and $95 \%$ air).

Cell proliferation assay. Cell proliferation was determined by the tetrazolium salt (MTT) method according to the manufacturer's instructions (Promega, Madison, WI). MAT-LyLu cells (2500 cells per well) were cultured in a 96well plate and were treated with different concentrations of GP $(0.0-4.0 \mu \mathrm{M})$ for 24,48 and $72 \mathrm{~h}$. At the end of the incubation period, tetrazolium was added, and colorimetric changes were measured by an ELISA plate reader at $570 \mathrm{~nm}$. A data point represents the mean \pm SD of one experiment repeated at least twice.

Thymidine incorporation assay. MAT-LyLu cells $\left(2 \times 10^{4}\right.$ cells per well) were cultured in 24-well plates. After $24 \mathrm{~h}$, MATLyLu cells were treated with different concentrations of TGFß1 $(0.0-10.0 \mathrm{ng} / \mathrm{ml})$ for $24 \mathrm{~h}$. The cells were then pulsed with $5 \mu \mathrm{Ci} / \mathrm{ml}$ of $\left[{ }^{3} \mathrm{H}\right]$-thymidine (NEN Corp., Boston, MA) for $3 \mathrm{~h}$. At the end of this period, the cells were washed twice with HBSS and fixed with methanol/acetic acid (3:1). The radioactivity of samples was counted on a B-counter as previously described (9). Amounts of $\left[{ }^{3} \mathrm{H}\right]$-thymidine incorporated into DNA were presented as dpm/well.
Colony formation (anchorage-independent growth). MATLyLu cells were harvested and plated on 6-well plates for colony formation assay. After 14 days, dishes were stained with $0.005 \%$ crystal violet, and the colonies were counted manually under a microscope.

Cell cycle analysis. MAT-LyLu cells were cultured in $75-\mathrm{cm}^{2}$ flasks at a density of $2 \times 10^{6}$ in $10 \mathrm{ml}$ of RPMI-1640 with $5 \%$ FCS for $24 \mathrm{~h}$ and then treated with the different concentrations of GP $(0.0-4.0 \mu \mathrm{M})$ for the indicated periods of time $(0-48 \mathrm{~h})$. After incubation, the cells were harvested, washed twice with HBSS containing 1\% FCS and resuspended in propidium iodine. Samples were analyzed on a coulter EPICS Elite flow cytometer (Hialeah, FL) as previously described (6). The fractions of cells in different phases of the cell cycle (G0/G1, S, G2/M) were presented as a percentage of total cells analyzed.

Reverse transcription-polymerase chain reaction (RT-PCR). MAT-LyLu cells were treated with different concentrations of GP (0.0-2.0 $\mu \mathrm{M})$ for $24 \mathrm{~h}$. The total RNA isolation, complementary DNA (cDNA) synthesis and PCR for TGFß1 and $\beta$-actin were performed as previously described (9). The final RT-PCR products $(10 \mu 1)$ were run on a $1.5 \%$ agarose gel containing ethidium bromide. The specific bands were quantified by ImageQuant software (Molecular Dynamics, Sunnyvale, CA). Results were presented as the ratio of TGFß1 to $ß$-actin.

ELISA for TGFß1 analysis. MAT-LyLu cells were cultured in 24-well culture plates and treated with GP (0.0-2.0 $\mu \mathrm{M})$ for $24 \mathrm{~h}$. At $24 \mathrm{~h}$ of incubation, the conditional media from cells were collected, and the secreted TGFß1 protein was measured as previously described (9). The TGFß1 concentration in the conditioned media was presented as $\mathrm{pg} / \mu \mathrm{g}$ cell protein. The total cell protein in each well was harvested and determined as previously described (9).

Western blot analysis. MAT-LyLu cells were treated with different concentrations of GP $(0.0-4.0 \mu \mathrm{M})$ for $24 \mathrm{~h}$, and cell extracts were prepared as previously described (9). Equal amounts of proteins (20 $\mu \mathrm{g} /$ lane $)$ were separated on $12 \%$ Tris-HCI gel (Bio-Rad Laboratories, Hercules, CA) and transferred to a PVDF membrane (Millipore, Bedford, MA). The membrane was incubated with the corresponding primary antibodies diluted 1:1000 in blocking solution, as indicated: a rabbit anti-phospho-Akt (ser473) polyclonal antibody (Cell Signaling Technology, Danvers, MA); a rabbit anti-Akt polyclonal antibody, a rabbit anti-cyclin D1 polyclonal antibody, a rabbit anti-cdk4 polyclonal antibody, a rabbit anti-p21 polyclonal antibody, a rabbit anti-phospho-Rb polyclonal antibody, a rabbit anti-Rb polyclonal antibody, and a mouse anti- $\beta$-actin monoclonal antibody (Santa Cruz Biotechnology, Santa Cruz, CA), respectively. Anti-mouse or anti-rabbit secondary antibodies (Amersham Biosciences, Buckinghamshire, UK) were used for antibody recognition. Antibody-bound proteins were detected by ECL Western Blotting Detection System (Amersham Biosciences).

Statistical analysis. Data were expressed as the mean \pm standard deviation (SD) of 4 culture wells. Statistical 
A

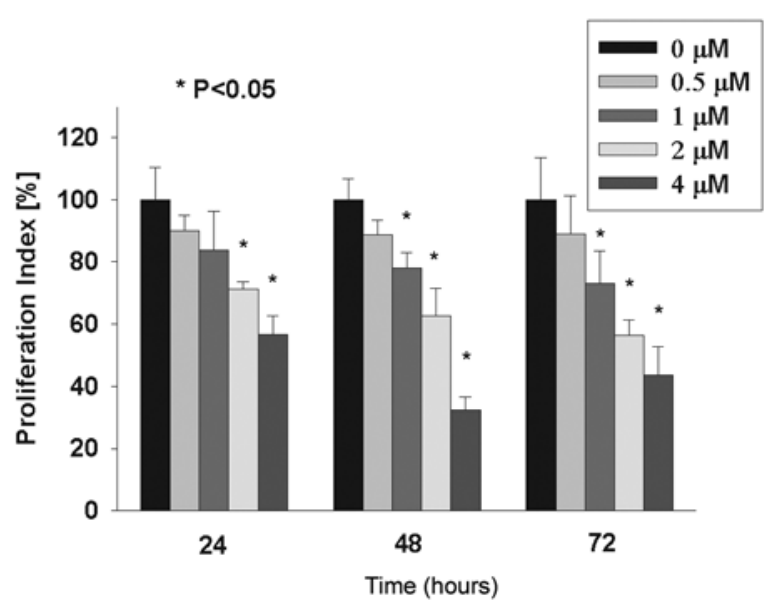

B

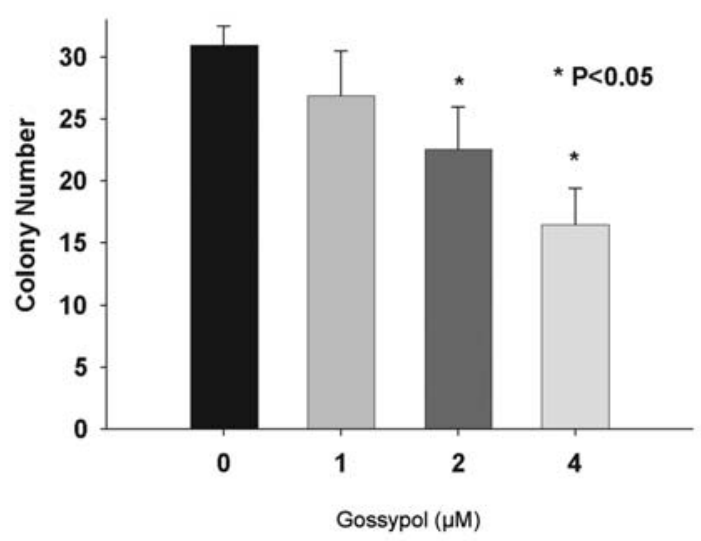

Figure 1. Effects of gossypol (GP) on the proliferation of MAT-LyLu cells. (A) MAT-LyLu cells were treated with GP $(0-4.0 \mu \mathrm{M})$. Cell proliferation was determined by the tetrazolium salt (MTT) method. (B) Anchorageindependent growth (colony formation) of MAT-LyLu cells was assessed on soft agarose containing GP $(0-4.0 \mu \mathrm{M})$ after incubation for 14 days. Each bar represents the mean \pm SD of 3 replicate samples. "Significantly different from the control group; $\mathrm{p}<0.05$.

differences between means were evaluated using one-way analysis of variance (ANOVA) followed by Tukey's pairwise comparisons. A probability (p) $<0.05$ was considered significant.

\section{Results}

Effects of GP on the proliferation of MAT-LyLu cells. In order to evaluate whether GP possesses an anti-proliferative property in the androgen-independent prostate cancer cells, we examined the effects of GP on the growth of MAT-LyLu. MAT-LyLu cells were treated with increasing concentrations of GP $(0.0,0.5,1.0,2.0$ and $4.0 \mu \mathrm{M})$ for 24,48 and $72 \mathrm{~h}$, and proliferation was determined by MTT assay. GP significantly inhibited the growth of MAT-LyLu cells as shown in Fig. 1A. At $24 \mathrm{~h}, \mathrm{GP}$ at the concentrations of 1.0, 2.0 and $4.0 \mu \mathrm{M}$ inhibited cell proliferation by $16.3,28.8$ and $43.4 \%$ respectively; at $48 \mathrm{~h}, \mathrm{GP}$ at the concentrations of $1.0,2.0$ and $4.0 \mu \mathrm{M}$ inhibited cell proliferation by $22.1,37.3$ and $67.5 \%$ respectively. The $\mathrm{IC}_{50}$ of GP for 48- and 72-h treatment was 2.64 and $1.25 \mu \mathrm{M}$, respectively. The results showed that GP inhibited the growth of MAT-LyLu cells in a dose- and
A

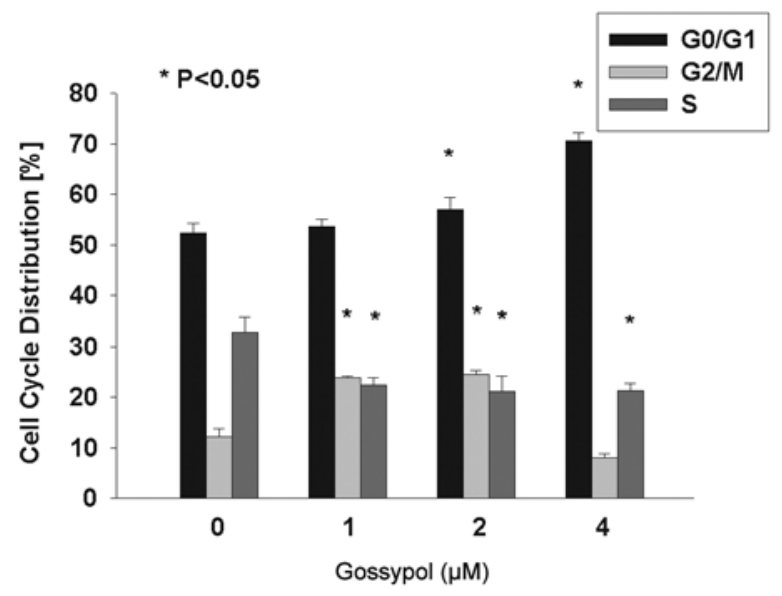

B

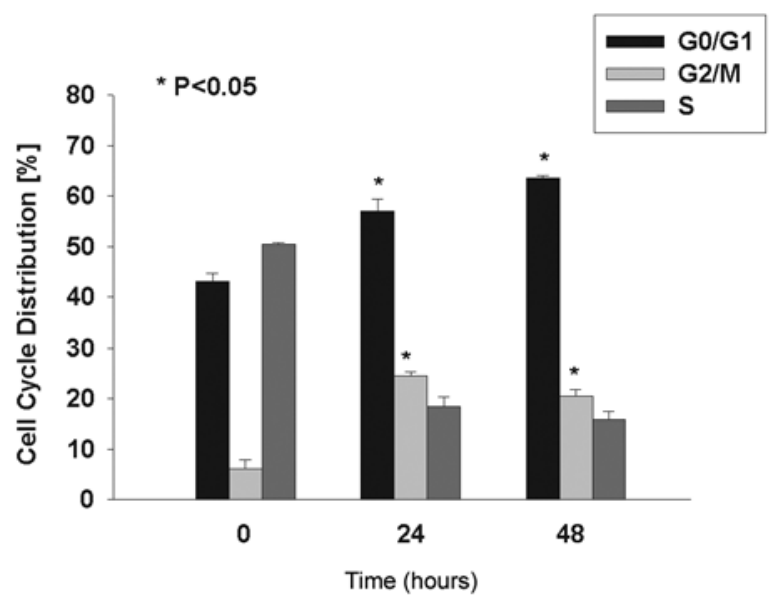

Figure 2. Effects of gossypol (GP) on the cell cycle distribution of MATLyLu cells. (A) MAT-LyLu cells were treated with GP $(0-4 \mu \mathrm{M})$ for $24 \mathrm{~h}$. The cell populations at G0/G1, S and G2/M phase were determined by flow cytometry. (B) MAT-LyLu cells were treated with GP $(2.0 \mu \mathrm{M})$ for 0,24 and $48 \mathrm{~h}$. The cell populations at G0/G1, S and G2/M phase were determined by flow cytometry. Each bar represents the mean \pm SD of 3 replicate samples. ${ }^{*}$ Significantly different from the control group; $\mathrm{p}<0.05$.

time-dependent manner. In order to test whether GP has inhibitory effects on the metastatic potential of prostate cancer cells, we performed colony formation assay in GP-treated MAT-LyLu cells. GP at the same concentrations $(0.0,1.0,20$ and $4.0 \mu \mathrm{M}$ ) decreased the number of cell colonies (Fig. 1B). These results indicate that GP inhibits the anchoragedependent (cell proliferation) and -independent growth (colony formation) of MAT-LyLu cells.

Effect of GP on the cell cycle distribution of MAT-LyLu cells. Since the inhibition of growth may be mediated by cell cycle growth arrest or apoptosis, we considered the possibility that the growth inhibition of MAT-LyLu cells induced by GP may be related to arrest at specific checkpoints in the cell cycle. To examine whether GP affects cell cycle distribution in MAT-LyLu cells, the cells were treated with different concentrations of GP $(0.0,1.0,2.0$ and $4.0 \mu \mathrm{M})$ for the indicated periods of time. Cell cycle distribution was performed by cytometric analysis. As shown in Fig. 2, GP increased the percentage of the cell population at $\mathrm{G} 0 / \mathrm{G} 1$ phase and decreased the percentage of the cell population in 
A

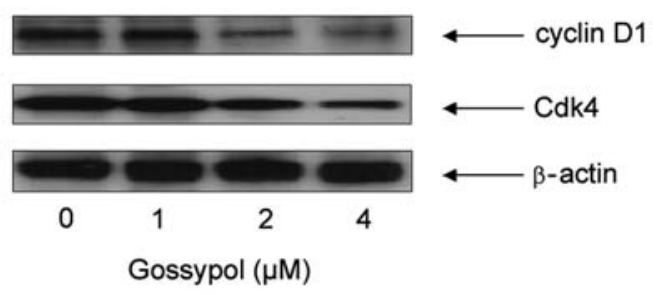

B

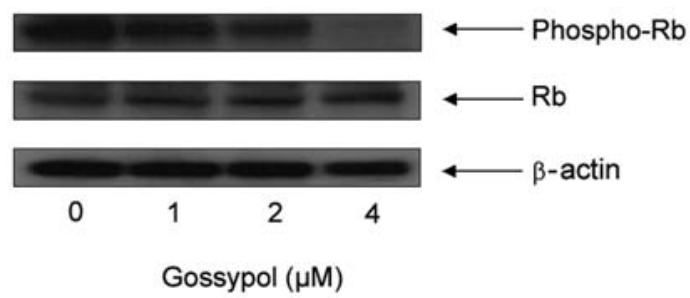

Figure 3. Effect of gossypol (GP) on expression of cell cycle regulatory proteins in MAT-LyLu cells. MAT-LyLu cells were treated with GP $(0-4.0 \mu \mathrm{M})$ for $24 \mathrm{~h}$, and whole cell extracts were subjected to Western blot analysis. (A) Expression of Cdk4 and cyclin D1 was measured by Western blot analysis with the corresponding antibodies. (B) Expression of phospho- $\mathrm{Rb}$ and $\mathrm{Rb}$ was performed by Western blot analysis with the corresponding antibodies. Equal protein loading was verified with anti- $\beta$-actin antibody as described in Materials and methods. Results are representative of three separate experiments.

$\mathrm{S}$ phase. The percentage of cells in G0/G1 phase increased from 52.4 to $70.6 \%$, while the percentage of cells in $\mathrm{S}$ phase decreased from 32.9 to $21.3 \%$ (Fig. 2A). Similarly, we also found that GP increased the percentage of cell population at G0/G1 phase in a time-dependent manner (Fig. 2B). These data showed that GP treatment resulted in cell cycle arrest of MAT-LyLu cells at G0/G1 phase in a dose- and timedependent manner. Although GP at 1.0 and $2.0 \mu \mathrm{M}$ caused an increase in the percentage of the cell population at $\mathrm{G} 2 / \mathrm{M}$ phase, we did not observe this effect at a high concentration of GP $(4.0 \mu \mathrm{M})$. Thus, the inhibitory effects of GP on MAT-LyLu cells may be due to cell cycle arrest at G0/G1 phase.

Effect of GP on the expression of cell cycle-regulated proteins in MAT-LyLu cells. The progression of cell cycle is controlled by cyclin and cyclin-dependent kinase. In order to determine how GP modulates the expression of cell cycle regulatory proteins involved in the control of G1/S phase transition of the cell cycle, we investigated the expression of cyclin D1 and Cdk4 in MAT-LyLu cells by Western blot analysis (Fig. 3A). GP resulted in a decrease in Cdk4 and cyclin D1 protein expression in MAT-LyLu cells, but did not result in appreciable change in expression of p21 protein (data not shown). Similarly, GP also decreased the expression of phospho-retinoblastoma $(\mathrm{Rb})$, but did not cause any significant change in the expression of $\mathrm{Rb}$ (Fig. 3B). Cancer cell growth arrest at G0/G1 phase by influencing the function of cyclin D1 and Cdk4 was also reported by other researchers $(23,24)$. These data suggest that the G0/G1 phase cell growth arrest in MAT-LyLu cells by GP is mediated through the downregulation of Cdk4 and cyclin D1, which further results in down-regulation of phosphorylation of $\mathrm{Rb}$ protein.
A
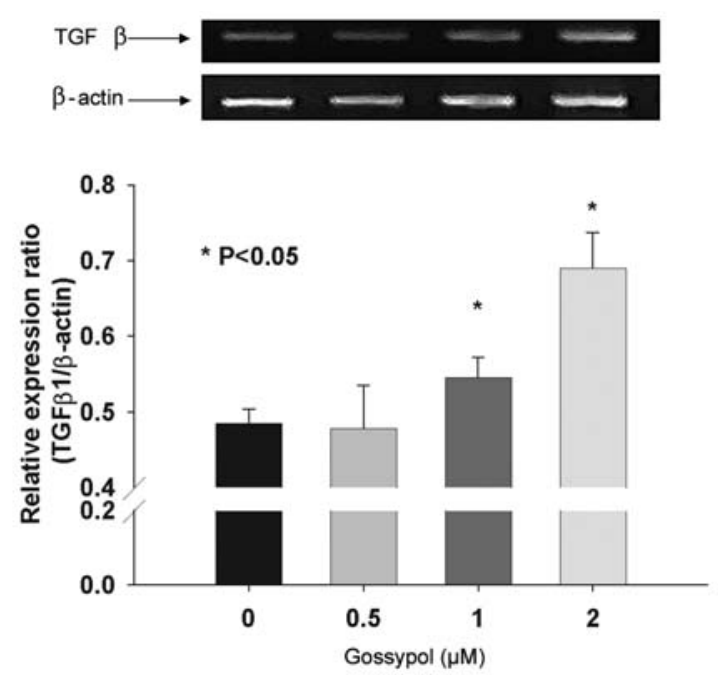

B

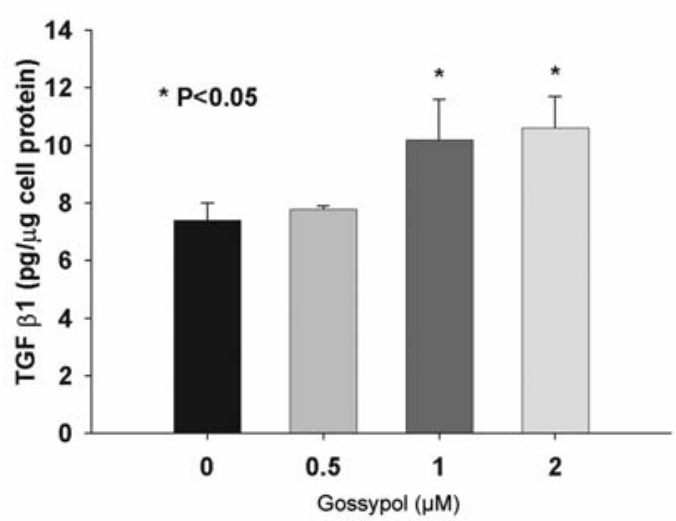

C

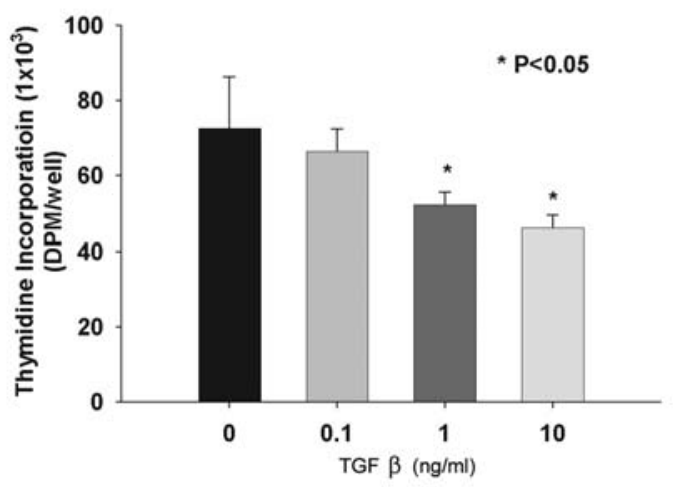

Figure 4. Effects of gossypol (GP) on mRNA expression and secretion of TGFß1 in MAT-LyLu cells. (A) mRNA expression of TGFß1 of MAT-LyLu cells was measured by RT-PCR analysis. Total RNA isolated from MAT-LyLu cells was used for RT-PCR analysis of TGFB1 mRNA expression. B-actin was used as an internal loading control. Results are expressed as the relative expression ratios of TGFß1 to B-actin. (B) MAT-LyLu cells were treated with different concentrations of GP $(0-2.0 \mu \mathrm{M})$ for $24 \mathrm{~h}$. At the end of this treatment period, TGFß1 secretion was measured as described in Materials and methods. (C) MAT-LyLu cells were treated with $0.0,0.1,1.0$ and $10 \mathrm{ng} / \mathrm{ml}$ of TGFß1 for $24 \mathrm{~h}$. Effects of TGFß1 on DNA synthesis of MAT-LyLu cells were assessed by thymidine incorporation assay. Each bar represents the mean \pm SD of 3 replicate samples. *Significantly different from the control group; $\mathrm{p}<0.05$.

Effect of GP on the mRNA expression and secretion of TGF $\beta 1$ in MAT-LyLu cells. The mechanism by which GP triggers an inhibitory pathway leading to growth inhibition of cultured 


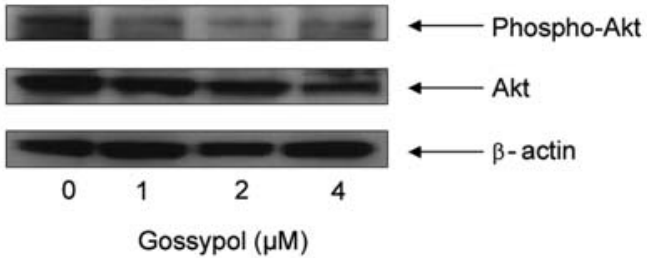

Figure 5. Effects of gossypol (GP) on expression of phospho-Akt and Akt in MAT-LyLu cells. MAT-LyLu cells were treated with GP $(0-4.0 \mu \mathrm{M})$ for $24 \mathrm{~h}$, and whole cell extracts were subjected to Western blot analysis. Expression of phospho-Akt (Ser473) and Akt was measured by Western blot analysis with the corresponding antibodies. Equal protein loading was verified with anti- $\beta$-actin antibody as described in Materials and methods. The results are representative of three separate experiments.

human prostate cancer PC-3 cells at G0/G1 phase of the cell cycle is known (6). However, how TGFß1 affects cell cycleregulated proteins in GP-treated prostate cancer cells remains unknown. In this study, we examined the effects of GP on mRNA expression and secretion of TGFß1 in MAT-LyLu cells. RT-PCR results showed that GP at 1.0 and $2.0 \mu \mathrm{M}$ resulted in a marked elevation of TGFß1 mRNA expression of MAT-LyLu cells, while the treatment of MAT-LyLu cells with $0.5 \mu \mathrm{M}$ of GP had no significant effect on the expression level of TGFß1 mRNA (Fig. 4A). As expected, GP treatment significantly enhanced TGFß1 secretions of MAT-LyLu cells in a dose-dependent manner $(\mathrm{p}<0.05)$. GP at $0.5,1.0$ and $2.0 \mu \mathrm{M}$ increased TGFß1 secretions by $1.05-, 1.38-$ and 1.43-fold compared to the control, respectively (Fig. 4B).

To confirm the ability of MAT-LyLu cells to respond to the inhibitory effects of exogenous TGFß1, the cells were treated with different concentrations of TGFB1 $(0,0.1,1.0$ and $10 \mathrm{ng} / \mathrm{ml}$ ) for $24 \mathrm{~h}$. Results showed that exogenous TGFß1 inhibited the growth of MAT-LyLu cells in a dose-dependent manner (Fig. 4C). TGFß1 (10 ng/ml) inhibited the DNA synthesis of MAT-LyLu cells by $63.8 \%$ compared to the control. Therefore, growth inhibition and G0/G1 phase cell cycle arrest in the GP-treated MAT-LyLu cells are mediated through the up-regulation of TGFß1 secretion.

Effect of GP on the expression of phospho-Akt and Akt in MAT-LyLu cells. Akt is a serine-threonine kinase which controls the activation of $\mathrm{NF}-\kappa \mathrm{B}$, which regulates expression and activity of cyclin D1 in breast and prostate cancer cells (25-28). We investigated whether the inhibitory effects of GP are mediated through the suppression of Akt kinase in prostate cancer cells, which results in the reduction of cyclin D1 expression. The cell extracts from MAT-LyLu cells treated with GP (0-4.0 $\mu \mathrm{M})$ were subjected to Western blot analysis. As seen in Fig. 5, GP resulted in the reduction in expression of Akt in MAT-LyLu cells in a dose-dependent manner. In addition, GP also markedly decreased phosphorylation of Akt at Ser473. Thus, cell cycle arrest at G0/G1 phase by GP is also the result of suppression of Akt activity, which results in down-regulation of cyclin D1 expression.

\section{Discussion}

We previously reported that GP up-regulated the expression of TGFß1, which resulted in G0/G1 cell cycle arrest of human prostate cancer and human BPH cells $(6,9,29)$. In this study, we investigated the molecular mechanism of GP-induced cell cycle in rodent prostate cancer cells. We showed that GP inhibited the anchorage-dependent (cell proliferation) and anchorage-independent growth (colony formation) in MATLyLu cells. Consistent with its ability to inhibit cell proliferation of MAT-LyLu cells, GP inhibited colony formation, stimulated mRNA expression and secretion of TGFß1, and inhibited Akt activity of the MAT-LyLu cells in a dosedependent manner. GP also inhibited the growth of prostate cancer cells by cell cycle arrest at G0/G1 phase. Although it has been reported that GP may arrest the cell cycle at S phase (30), our results revealed that GP might result in an accumulation of the cell population at $\mathrm{G} 0 / \mathrm{G} 1$ phase of the cell cycle with a decrease in the cell population at $\mathrm{S}$ phase. Our observation that GP induces cell cycle arrest at G0/G1 phase in MAT-LyLu cells is in accordance with our previous study demonstrating cell cycle arrest at G0/G1 phase in PC-3 (6) and human BPH cells (29). It is reasonable to assume that GP inhibits the growth of MAT-LyLu cells through cell cycle arrest at $\mathrm{G} 0 / \mathrm{G} 1$ phase.

Since aberrantly active cell cycle regulatory proteins such as cyclin D1 and cyclin-dependent kinase (Cdk4) are responsible for the uncontrolled growth of cancer cells (31), these proteins are suitable therapeutic targets. Ligueros et al demonstrated that GP arrested the cell cycle at G1/S phase by decreasing $\mathrm{Rb}$ expression, $\mathrm{Rb}$ phosphorylation, and cycle D1 protein expression in human MCF-7 breast cancer cells (8). Thus, we aimed to determine whether GP affects the expression of cell cycle-regulated proteins, such as $\mathrm{Cdk} 4$, cyclin D1, Rb and phospho-Rb in MAT-LyLu cells. We evaluated the influences of GP on the expression of cell cycle regulators such as cyclin D1, Cdk4 and Rb, which regulate cell cycle progression. As shown in Fig. 3A, GP markedly decreased cyclin D1 expression in a dose-dependent manner. Since cyclin D1 regulates the activity of cyclin-dependent kinase Cdk4, which controls the G1 cell cycle checkpoint, we investigated the effects of GP on Cdk4 kinase. As expected, GP also inhibited expression of Cdk4 suggesting that cell cycle arrest at G0/G1 phase by GP is a result of inhibition of cell cycle regulatory proteins such as cyclin D1 and Cdk4.

Since cyclin D1 forms a complex with Cdk4/Cdk6 to further phosphorylate $\mathrm{Rb}$ with the release of general transcription factor E2F1 which accelerates cell cycle progression from $\mathrm{G} 1$ to $\mathrm{S}$ phase (31), we subsequently examined the effects of GP on expression of phosphorylated $\mathrm{Rb}$ and total $\mathrm{Rb}$ in MAT-LyLu cells. GP suppressed the phosphorylation of $\mathrm{Rb}$, but did not cause an obvious change in the level of expression of total $\mathrm{Rb}$ (Fig. 3B). Thus, the anti-proliferative effects were mediated through the down-regulation of expression of Cdk4 and cyclin D1, leading to the inhibition of phosphorylation of $\mathrm{Rb}$ and cell cycle arrest at $\mathrm{G} 0 / \mathrm{G} 1$ phase. This observation is in agreement with our previous study demonstrating cell cycle arrest at G1/G0 phase of prostate cancers and down-regulation of cyclin D1 by GP $(6,9)$. One clinical study showed that GP decreased cyclin D1 expression and increased the nuclear $\mathrm{Rb}$ expression in breast cancer patients (7). Huang et al showed that (-)-GP inhibited the growth of prostate cancer DU-145 cells through up-regulation of p21 and down-regulation of $\mathrm{Cdk} 4$, cyclin D1 and $\mathrm{Rb}$ 
proteins (10). Although we found inhibitory effects of GP on the expression of cyclin D1, Cdk4 and phospho-Rb in MATLyLu cells, we did not find any inhibitory effect of GP on the expression of total $\mathrm{Rb}$ and $\mathrm{p} 21$ (data not shown) in these cells.

TGFß1 is a potent growth inhibitor in most epithelial cells including cancer cells. TGFß1 mediates growth inhibition by blocking cell cycle progression from $\mathrm{G} 1$ to $\mathrm{S}$ phase. In most cases, it induces cell cycle arrest at G0/G1 phase via preventing the active cyclin D1-Cdk4 complex and $\mathrm{Rb}$ phosphorylation $(21,32)$. TGFß1 has been shown to decrease cyclin D1 expression in rat intestinal epithelial cells (33), expression of $\mathrm{Rb}$ and phosphorylated $\mathrm{Rb}$ in Mv1Lu cells (34), and expression of c-Myc in keratinocytes (21). We previously showed that cell cycle arrest at G0/G1 phase of human prostate cancer and human BPH cells are mediated through stimulation of TGFß1 expression $(6,9,29)$. It is possible that inhibition of expression of cyclin D1 and Cdk4 by GP could be mediated by up-regulation of TGFß1 secretion. In the present study, we demonstrated the stimulatory effects of GP on mRNA expression and secretion of TGFß1 in MAT-LyLu cells. Moreover, the stimulatory effect of GP on secretion of TGFß1 correlates well with its ability to arrest the cell cycle at G0/ G1 phase. The results that MAT-LyLu cells secreted and responded to TGFß1 suggest that TGFß1 serves as a negative regulator for MAT-LyLu cells. These findings, along with the observations that GP reduced the expression of cyclin D1, Cdk4 and phospho-Rb protein of MAT-LyLu cells, suggest that the anti-proliferative activity of GP is mediated by inducing TGFß1 protein production in MAT-LyLu cells, which further regulates the function of cyclin D1, Cdk4 and phospho-Rb protein in cell cycle progression. Our data suggest that GP probably exerts its effect at the transcriptional level within the cell cycle regulatory pathway.

Akt is a family of serine/threonine kinases and consists of three highly conserved kinases; Akt1, Akt2, Akt3. Elevated gene expression and kinase activities of Akt have been observed in many human cancers including prostate cancer (35), suggesting that Akt may play an important role in the development and progression of malignancy. Because Akt kinase controls the activation of NF- $\mathrm{KB}$ (25) and expression of NF- $\kappa$ B-regulated cyclin D1 (26), the suppression of cyclin D1 expression might be mediated by the inhibition of Akt kinase activity. A recent study showed that TGFß1 treatment decreased phosphorylation of Akt at Ser473 in human colon cancer cells (22). This prompted us to hypothesize that GP may inhibit Akt phosphorylation directly or indirectly by modulating TGFß1 signaling. In order to test this hypothesis, we examined the effects of GP on expression of Akt and phospho-Akt in MAT-LyLu cells. We demonstrated that GP suppresses the expression of Akt and activity of Akt by inhibiting phosphorylation of Akt at Ser473. Notably, isoflavone and genistein, which inhibit Akt phosphorylation, have been shown to inhibit expression of cyclin D1 in dorsolateral prostate tissues of TRAMP/FVB mice (36). Collectively, we conclude that GP induces cell cycle arrest at G0/G1 phase in MAT-LyLu cells by inhibition of Akt kinase activity, which suppresses expression of cyclin D1 and Cdk4 kinase leading to a decrease in the phosphorylation of $\mathrm{Rb}$. Our results further confirm the role of Akt as an oncogene responsible for the growth and survival of cancer cells. Li et al demonstrated that the Akt pathway is a major survival pathway in human cancer, and the inhibition of Akt may suppress the activity of regulatory proteins of $\mathrm{G} 1 / \mathrm{S}$ cell cycle progression, such as cyclin D1 and Rb (35).

In conclusion, we demonstrated that GP inhibited Akt, stimulated the expression and secretion of TGFß1, and inhibited expression of cyclin D1 and phospho-Rb in prostate cancer cells. The inhibitory effects of GP on MAT-LyLu cells are mediated by up-regulating TGFß1 secretion and downregulating phosphorylation and expression of Akt kinase, which suppresses expression of cell cycle regulatory proteins cyclin D1, Cdk4 and phospho-Rb, leading to cell cycle arrest at G0/G1 phase and growth inhibition. Thus, GP exerts its effects on cancer cells by multiple mechanisms and may have potential therapeutic use for the treatment of prostate cancer.

\section{Acknowledgements}

This study was partially supported by NIH grants CA66193 and P30CA16058.

\section{References}

1. Jemal A, Siegel R, Ward E, Murray T, Xu J and Thun MJ: Cancer statistics, 2007. CA Cancer J Clin 57: 43-66, 2007.

2. Isaacs JT: Prostatic cancer: an age-old problem. Adv Exp Med Biol 330: 167-184, 1993.

3. Nessler-Menardi C, Jotova I, Culig Z, Eder IE, Putz T, Bartsch G and Klocker $\mathrm{H}$ : Expression of androgen receptor coregulatory proteins in prostate cancer and stromal-cell culture models. Prostate 45: 124-131, 2000.

4. Zhou JR, Yu L, Zerbini LF, Libermann TA and Blackburn GL: Progression to androgen-independent $\mathrm{LNCaP}$ human prostate tumors: cellular and molecular alterations. Int J Cancer 110: 800-806, 2004.

5. Chang CJ, Ghosh PK, Hu YF, Brueggemeier RW and Lin YC: Antiproliferative and antimetastatic effects of gossypol on Dunning prostate cell-bearing Copenhagen rats. Res Commun Chem Pathol Pharmacol 79: 293-312, 1993.

6. Shidaifat F, Canatan H, Kulp SK, Sugimoto Y, Chang WY, Zhang Y, Brueggemeier RW, Somers WJ and Lin YC: Inhibition of human prostate cancer cell growth by gossypol is associated with stimulation of transforming growth factor-beta. Cancer Lett 107: 37-44, 1996.

7. Van Poznak C, Seidman AD, Reidenberg MM, Moasser MM, Sklarin N, Van Zee K, Borgen P, Gollub M, Bacotti D, Yao TJ, Bloch R, Ligueros M, Sonenberg M, Norton L and Hudis C: Oral gossypol in the treatment of patients with refractory metastatic breast cancer: a phase I/II clinical trial. Breast Cancer Res Treat 66: 239-248, 2001

8. Ligueros M, Jeoung D, Tang B, Hochhauser D, Reidenberg MM and Sonenberg M: Gossypol inhibition of mitosis, cyclin D1 and $\mathrm{Rb}$ protein in human mammary cancer cells and cyclin-D1 transfected human fibrosarcoma cells. Br J Cancer 76: 21-28, 1997.

9. Jiang J, Sugimoto Y, Liu S, Chang HL, Park KY, Kulp SK and Lin YC: The inhibitory effects of gossypol on human prostate cancer cells-PC3 are associated with transforming growth factor beta1 (TGFbeta1) signal transduction pathway. Anticancer Res 24: 91-100, 2004

10. Huang YW, Wang LS, Chang HL, Ye W, Sugimoto Y, Dowd MK, Wan PJ and Lin YC: Effects of serum on (-)-gossypolsuppressed growth in human prostate cancer cells. Anticancer Res 26: 3613-3620, 2006.

11. Zhang M, Liu H, Tian Z, Griffith BN, Ji M and Li QQ: Gossypol induces apoptosis in human PC-3 prostate cancer cells by modulating caspase-dependent and caspase-independent cell death pathways. Life Sci 80: 767-774, 2007.

12. Zhang M, Liu H, Guo R, Ling Y, Wu X, Li B, Roller PP, Wang S and Yang D: Molecular mechanism of gossypol-induced cell growth inhibition and cell death of HT-29 human colon carcinoma cells. Biochem Pharmacol 66: 93-103, 2003. 
13. Liu S, Kulp SK, Sugimoto Y, Jiang J, Chang HL, Dowd MK, Wan P and Lin YC: The (-)-enantiomer of gossypol possesses higher anticancer potency than racemic gossypol in human breast cancer. Anticancer Res 22: 33-38, 2002.

14. Huang YW, Wang LS, Chang HL, Ye W, Dowd MK, Wan PJ and Lin YC: Molecular mechanisms of (-)-gossypol-induced apoptosis in human prostate cancer cells. Anticancer Res 26 1925-1933, 2006.

15. Xu L, Yang D, Wang S, Tang W, Liu M, Davis M, Chen J, Rae JM, Lawrence T and Lippman ME: (-)-Gossypol enhances response to radiation therapy and results in tumor regression of human prostate cancer. Mol Cancer Ther 4: 197-205, 2005.

16. Lei X, Chen Y, Du G, Yu W, Wang X, Qu H, Xia B, He H, Mao J, Zong W, Liao X, Mehrpour M, Hao X and Chen Q: Gossypol induces Bax/Bak-independent activation of apoptosis and cytochrome $\mathrm{c}$ release via a conformational change in $\mathrm{Bcl}-2$. FASEB J 20: 2147-2149, 2006.

17. Koli K and Keski-Oja J: 1,25-Dihydroxyvitamin D3 enhances the expression of transforming growth factor beta 1 and its latent form binding protein in cultured breast carcinoma cells. Cancer Res 55: 1540-1546, 1995.

18. Yu Z, Tang Y, Hu D and Li J: Inhibitory effect of genistein on mouse colon cancer MC-26 cells involved TGF-beta1/Smad pathway. Biochem Biophys Res Commun 333: 827-832, 2005.

19. Benson JR, Wakefield LM, Baum M and Colletta AA: Synthesis and secretion of transforming growth factor beta isoforms by primary cultures of human breast tumour fibroblasts in vitro and their modulation by tamoxifen. Br J Cancer 74: 352-358, 1996

20. Attisano L and Wrana JL: Signal transduction by the TGF-beta superfamily. Science 296: 1646-1647, 2002.

21. Ravitz MJ and Wenner CE: Cyclin-dependent kinase regulation during G1 phase and cell cycle regulation by TGF-beta. Adv Cancer Res 71: 165-207, 1997.

22. Wang J, Yang L, Yang J, Kuropatwinski K, Wang W, Liu XQ, Hauser J and Brattain MG: Transforming growth factor beta induces apoptosis through repressing the phosphoinositide 3kinase/AKT/survivin pathway in colon cancer cells. Cancer Res 68: 3152-3160, 2008.

23. Gupta S, Hussain T and Mukhtar H: Molecular pathway for (-)-epigallocatechin-3-gallate-induced cell cycle arrest and apoptosis of human prostate carcinoma cells. Arch Biochem Biophys 410: 177-185, 2003.

24. Huang X, Zhu D and Lou Y: A novel anticancer agent, icaritin induced cell growth inhibition, G1 arrest and mitochondrial transmembrane potential drop in human prostate carcinoma PC-3 cells. Eur J Pharmacol 564: 26-36, 2007.
25. Ozes ON, Mayo LD, Gustin JA, Pfeffer SR, Pfeffer LM and Donner DB: NF-kappaB activation by tumour necrosis factor requires the Akt serine-threonine kinase. Nature 401: 82-85, 1999.

26. Suh J and Rabson AB: NF-kappaB activation in human prostate cancer: important mediator or epiphenomenon? J Cell Biochem 91: 100-117, 2004.

27. Shukla S and Gupta S: Molecular mechanisms for apigenininduced cell-cycle arrest and apoptosis of hormone refractory human prostate carcinoma DU145 cells. Mol Carcinog 39: 114-126, 2004.

28. Jiang J, Slivova V, Harvey K, Valachovicova T and Sliva D: Ganoderma lucidum suppresses growth of breast cancer cells through the inhibition of Akt/NF-kappaB signaling. Nutr Cancer 49: 209-216, 2004.

29. Shidaifat F, Canatan H, Kulp SK, Sugimoto Y, Zhang Y, Brueggemeier RW, Somers WJ, Chang WY, Wang HC and Lin YC: Gossypol arrests human benign prostatic hyperplastic cell growth at G0/G1 phase of the cell cycle. Anticancer Res 17: 1003-1009, 1997.

30. Wang Y and Rao PN: Effect of gossypol on DNA synthesis and cell cycle progression of mammalian cells in vitro. Cancer Res 44: 35-38, 1984.

31. Takahashi-Yanaga F and Sasaguri T: GSK-3beta regulates cyclin D1 expression: a new target for chemotherapy. Cell Signal 20: 581-589, 2008.

32. Lee KY and Bae SC: TGF-beta-dependent cell growth arrest and apoptosis. J Biochem Mol Biol 35: 47-53, 2002.

33. Ko TC, Sheng HM, Reisman D, Thompson EA and Beauchamp RD: Transforming growth factor-beta 1 inhibits cyclin D1 expression in intestinal epithelial cells. Oncogene 10: 177-184, 1995.

34. Schwarz JK, Bassing CH, Kovesdi I, Datto MB, Blazing M, George S, Wang XF and Nevins JR: Expression of the E2F1 transcription factor overcomes type beta transforming growth factor-mediated growth suppression. Proc Natl Acad Sci USA 92: 483-487, 1995

35. Li L, Ittmann MM, Ayala G, Tsai MJ, Amato RJ, Wheeler TM, Miles BJ, Kadmon D and Thompson TC: The emerging role of the PI3-K-Akt pathway in prostate cancer progression. Prostate Cancer Prostatic Dis 8: 108-118, 2005.

36. El Touny LH and Banerjee PP: Akt GSK-3 pathway as a target in genistein-induced inhibition of TRAMP prostate cancer progression toward a poorly differentiated phenotype. Carcinogenesis 28: 1710-1717, 2007. 